> La génomique et la génétique sont en train de devenir des piliers de la recherche clinique permettant de comprendre certaines maladies au niveau moléculaire et d'améliorer les traitements. Cependant, la réalisation d'analyses génomiques dans le cadre d'études cliniques en oncologie soulève un certain nombre de problèmes liés aux procédés de recueil des échantillons, à la nécessité de procéder à des étapes pré-analytiques sur les sites cliniques et à la fragilité des molécules à analyser. La mise en place d'une logistique adaptée à l'identification et la validation de biomarqueurs issus des technologies génomiques nécessite de maîtriser les technologies utilisées en aval et requiert une intégration forte de tous les acteurs impliqués: centre de soins, sociétés de biotechnologies et/ou industrie pharmaceutique. <

\section{Place des analyses génomiques \\ centralisées dans \\ la découverte \\ et la validation \\ d'innovations \\ diagnostiques en \\ oncologie}

\section{Agnès Groulet-Martinec}

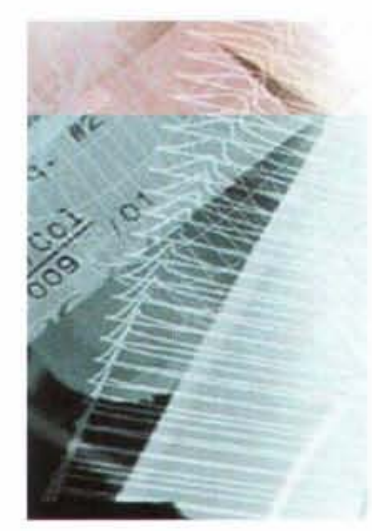

Chef de Projet, Ipsogen, Luminy Biotech Entreprises, Case 923, 163, avenue de Luminy, 13288 Marseille Cedex 9, France. martinec@ipsogen.com
L'apport de la génomique et de la génétique en recherche clinique est essentiel pour la compréhension des mécanismes moléculaires de certaines maladies et I'amélioration des traitements.

Les cancers par exemple apparaissent quand des gènes mutés ou défectueux activent ou bloquent de façon inappropriée une voie d'activation moléculaire cruciale pour une fonction biologique. La possibilité de détecter une mutation ou ses conséquences au niveau fonctionnel et la capacité de comprendre comment cette mutation contribue au développement de la maladie ou à la réponse à un traitement est un enjeu majeur pour améliorer la prise en charge des patients [1].

La génomique est l'étude d'ensemble de gènes, de leur polymorphisme, de leur expression et leur fonction dans un organisme donné. Une forme classique d'analyse génomique consiste à mesurer le niveau d'expression des gènes, autrement dit à mesurer la représentativité d'une ou plusieurs molécules d'ARN dans une cellule ou un tissu donné par analyse sur puces à $A D N$ ou RT-PCR quantitative. En fonction des échantillons étudiés, le niveau d'expression des gènes peut ainsi être corrélé au devenir clinique du patient ou encore à la réponse à un traitement donné.

De façon parallèle, le criblage systématique de mutations somatiques par génotypage de l'ADN des cellules tumorales permet d'acquérir un certain nombre d'informations sur les variations génétiques liées aux caractéristiques de la maladie ainsi qu'à sa sensibilité ou sa résistance aux traitements disponibles [2].

Les avancées technologiques en matière de génomique ont permis d'augmenter considérablement le nombre de gènes analysables tout en réduisant les coûts d'analyse, ouvrant ainsi les portes pour une utilisation clinique. L'accès à ces nouvelles technologies permet au médecin de mieux caractériser la maladie au niveau moléculaire, de manière spécifique pour chaque patient, et donc d'aider au diagnostic et au choix du traitement approprié (Figure 1).

Nous proposons dans cet article de décrire brièvement les différentes étapes du développement de ce type de test et de souligner les différents points critiques liés à l'utilisation de ressources biologiques pour des analyses génétique ou génomique dans le cadre d'essais multicentriques. 


\section{Développement d'un test de diagnostic}

Le développement d'un test de diagnostic à partir d'études de génomique se découpe en 3 phases (phase de découverte, phase de développement et phase clinique proprement dite) et fait appel à plusieurs types de ressources biologiques (Figure2) $[3,4]$.

\section{Phase de découverte}

Cette phase est conduite en collaboration avec des équipes académiques et des centres de soins. Le but principal de cette première série d'études est d'établir la preuve de concept à partir d'une collection d'échantillons et de démontrer la faisabilité du projet sur des échantillons de tumeurs archivés dans les tumorothèques, pour lesquels on dispose d'annotations cliniques; autrement dit, d'identifier des gènes ou des groupes de gènes pour lesquels la variation du niveau d'expression ou le niveau des altérations somatiques est corrélée au pronostic ou à la réponse à un traitement. Différentes études sur les lymphomes, le cancer du sein, les leucémies et le cancer du côlon ont ainsi été publiées par des équipes de l'Institut Paoli-Calmettes en collaboration avec Ipsogen [5-8].

\section{Phase de développement}

Cette étape doit permettre notamment de redéfinir le jeu de gènes identifiés précédemment (phase de découverte), de confirmer les performances de discrimination de ces marqueurs pour une population donnée de patients, et d'estimer les critères de sensibilité (taux de faux négatifs) et de spécificité (taux de faux positifs) de cette com- binaison de gènes. C'est le cas pour notre test sur le cancer du sein pour lequel des gènes corrélés au statut ERBB2 des tumeurs ont été identifiés, constituant une signature phénotypique dont les performances ont été comparées à celles des tests existants (IHC et FISH) [9].

À l'issue de cette phase, un véritable prototype de «test de diagnostic » est obtenu. C'est également au cours de cette phase que se fait la mise au point des aspects techniques liés au traitement des échantillons, au développement de contrôles de qualités etc., avec pour objectif de mettre en place un procédé reproductible le plus simple possible.

Un des problèmes liés aux échantillons archivés utilisés dans les études rétrospectives est l'absence de randomisation des échantillons, et donc la difficulté d'étendre la validité des marqueurs identifiés à la population générale.

\section{Phase de validation clinique}

II s'agit d'une phase de validation des résultats sur un ou plusieurs jeux de données indépendants. Dans une première série d'études cliniques, le test est appliqué dans le cadre d'un protocole d'essais de diagnostic. Dans ce cas, il s'agit toujours d'études rétrospectives mais sur des échantillons archivés (tumeurs congelées ou tissus fixés inclus en paraffine) et sélectionnés sans

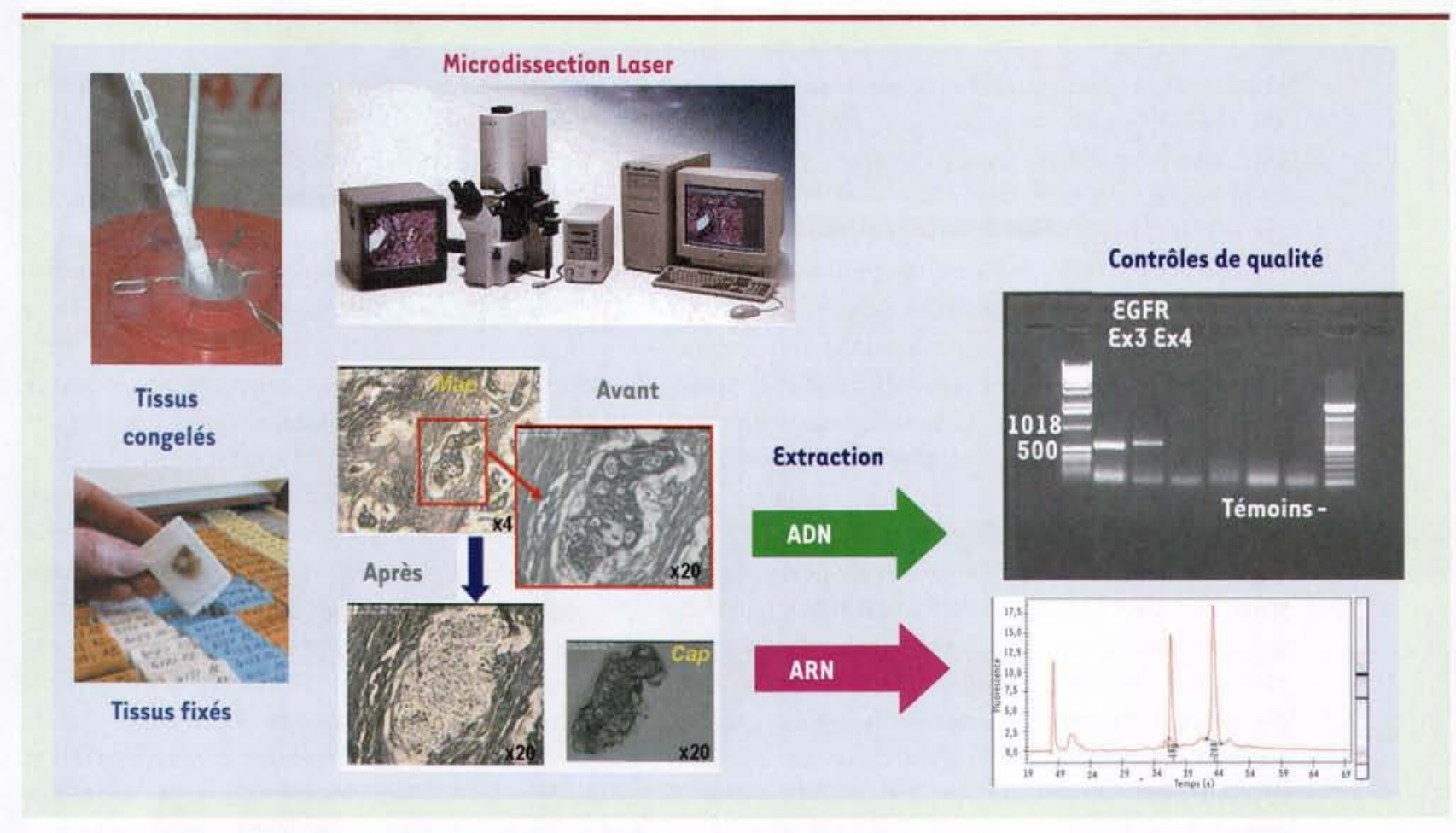

Figure 1. Exploitation des ressources biologiques en génomique. 
a priori pour la question posée. Ces échantillons (tests et références) archivés préférentiellement au cours d'études cliniques antérieures présentent l'avantage d'accéder à plusieurs années de recul clinique; la validation est de fait plus rapide que si l'on utilise des tissus frais issus de patients nouvellement diagnostiqués. L'objectif consiste dans ce cas à appliquer prospectivement et en aveugle le test clinique développé, à valider la valeur pronostique ou prédictive du test et en préciser les performances (spécificité, sensibilité, reproductibilité). La question posée est donc une question prospective qui interroge des échantillons archivés. Cette phase sera avantageusement réalisée en mode multicentrique afin de valider et standardiser les procédés de collecte, de stockage et de traitement des échantillons.

\section{Deuxième série d'études cliniques}

Dans cette deuxième série, le test est appliqué formellement à une large cohorte de patients dans le cadre d'un protocole d'essais cliniques pour guider la prescription. Ces études ont pour but de valider le test dans le cadre de son utilisation réelle en tenant compte des contraintes techniques de rendu de résultats, de recrutement et de pratiques spécifiques des centres de soins impliqués.
Elles ont également pour objectif de déterminer les performances cliniques du test dans un contexte où les biais sont rigoureusement contrôlés, et surtout d'en mesurer les bénéfices et les limites par rapport à une situation antérieure sans l'information nouvelle apportée par celui-ci. II est à noter que cette étape n'est pas obligatoire pour un enregistrement $C \varepsilon$ ou FDA d'un test diagnostique [10].

Toutes ces études requièrent l'utilisation d'un large nombre d'échantillons rigoureusement annotés qui font appel aux ressources de différents centres anticancéreux nationaux ou internationaux.

La réalisation d'analyses génomiques dans le cadre d'études cliniques en oncologie soulèvent un certain nombre de problèmes liés aux procédés de recueil des échantillons (chirurgie, biopsies invasives...), à la nécessité de procéder à des étapes pré-analytiques sur les sites cliniques et à la fragilité des molécules à analyser. Ce type d'analyses se distingue donc sensiblement des analyses biologiques couramment réalisées dans les études cliniques classiques.

Selon qu'il s'agit d'études rétrospectives ou prospectives, selon que I'on a accès à des prélèvements congelés ou à des tissus fixés inclus en paraffine, les contraintes de délai, de transport, de stockage et de traitement des échantillons sont différentes [11].

Nous avons donc mis en place une série de procédures standardisées et sécurisées ainsi que des contrôles de qualité pour assurer une bonne identification des prélèvements, la préservation et la traçabilité complète depuis les centres de soins jusqu'au rendu des résultats en prenant en compte les différentes contraintes liées à la nature des échantillons et au type d'étude.

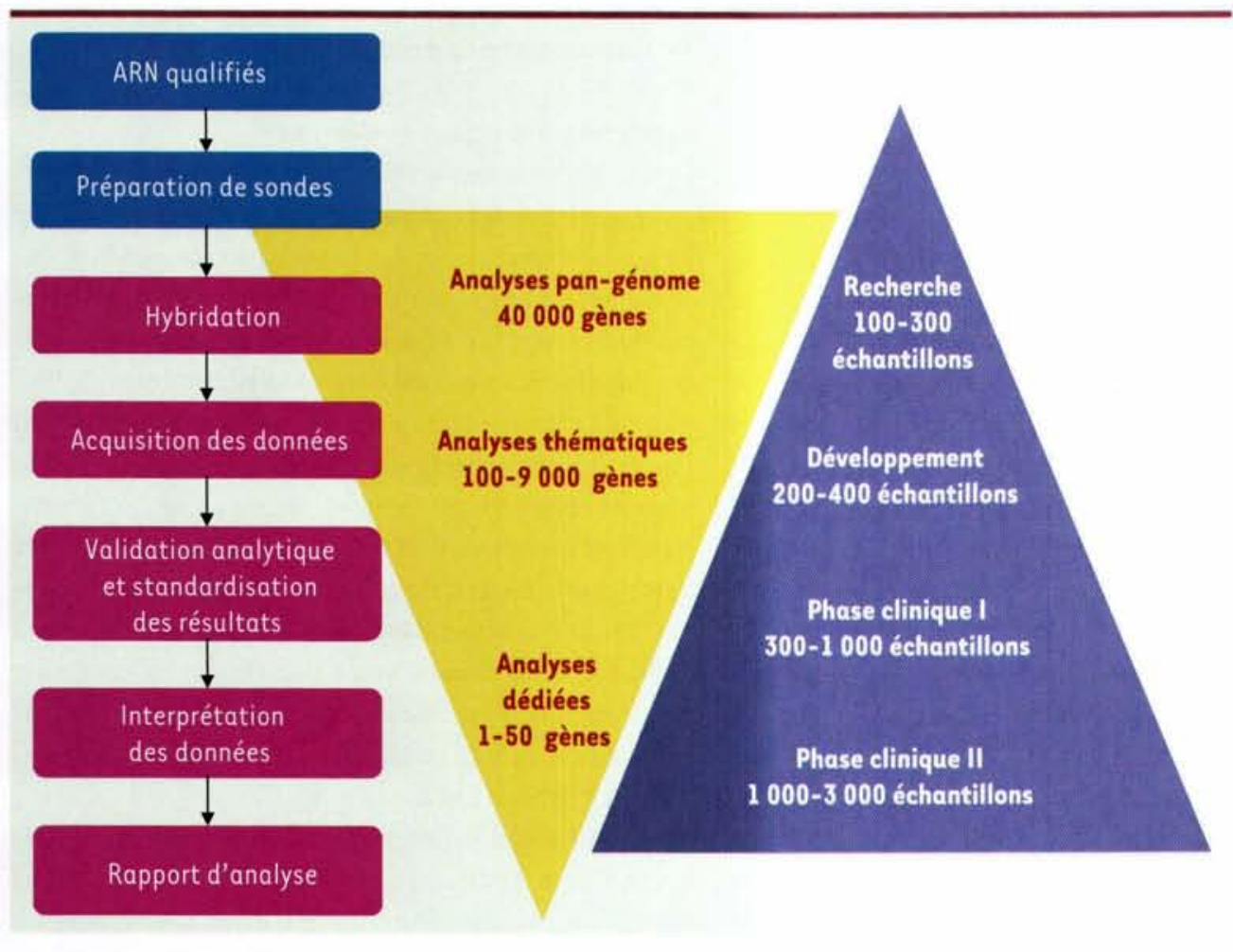

Figure 2. Les différentes phases de développement d'un test diagnostique.

\section{Recommandations}

Le recueil

et l'acheminement

des prélèvements

Pour les prélèvements destinés à des analyses moléculaires, cette étape est cruciale pour la qualité des résultats de l'étude. Elle requiert une importante motivation de l'ensemble des acteurs impliqués (cliniciens, anatomo-pathologistes, personnel des tumorothèques) pour éviter une ischémie de l'échantillon de trop longue durée et empêcher une dégradation des acides nucléiques, en particulier des ARN. Le délai entre le recueil du prélèvement et son conditionnement doit 
être le plus court possible (idéalement inférieur à 30 minutes). Chaque prélèvement est donc accompagné d'une feuille à la date du jour, comportant l'heure de l'exérèse chirurgicale, de la biopsie ou de la ponction, ainsi que l'heure d'arrivée au laboratoire.

\section{Le conditionnement des échantillons}

Les prélèvements peuvent être congelés ou fixés et inclus en paraffine. La congélation doit se faire le plus rapidement possible par immersion dans l'azote liquide $\left(-196^{\circ} \mathrm{C}\right)$, la chaîne du froid ne devant jamais être interrompue jusqu'à l'utilisation finale du prélèvement (conservation dans l'azote liquide ou dans un congélateur à $-80^{\circ} \mathrm{C}$ ) [11].

La fixation est l'étape la plus importante pour l'inclusion des tissus en paraffine. Nous avons donc validé des conditions de fixation (type de fixateur, température, durée, $\mathrm{pH}$, volume, taille de l'échantillon...) pour préserver l'intégrité des ADN et des ARN. Nous préconisons par exemple l'utilisation de fixateurs spécifiques et nous recommandons de ne pas dépasser un certain temps de fixation. L'intégrité des acides nucléiques est particulièrement remise en cause pour les spécimens archivés depuis plus de 10 ans.

Une troisième alternative consiste à préserver les tissus à température ambiante ( 1 semaine) ou à $4^{\circ} \mathrm{C}$ ( 1 mois) dans un conservateur tel que le RNALater ${ }^{\text {TM }}$ (Ambion).

Quel que soit le type de conditionnement retenu, cette étape est cruciale pour les analyses sur ADN et ARN. La rapidité et la rigueur nécessaires pour garantir la préservation des acides nucléiques se heurtent dans la plupart des cas à l'éloignement entre les blocs opératoires et les laboratoires d'anatomo-pathologie dans les hôpitaux. C'est pourquoi nous avons entrepris un travail de sensibilisation et mis en place des procédures standardisées ainsi que des feuilles de suivi des prélèvements pour garantir la bonne réalisation des analyses de génomique ou de génétique.

\section{L'identification des échantillons}

Conformément à la réglementation en vigueur en France, aucune donnée nominative ne doit transiter dans les laboratoires non habilités à les traiter. Nous avons donc mis en place l'attribution d'un identifiant unique pour chaque prélèvement dès la sortie des centres de soins après que nous nous soyons assurés que le formulaire de consentement éclairé a été préalablement signé par le patient. Ce numéro unique suit le prélèvement depuis son conditionnement jusqu'à l'analyse des données garantissant une traçabilité complète du processus sans perte d'information.

Le rapatriement des prélèvements des centres de soins vers Ipsogen Afin de sécuriser le rapatriement des prélèvements des centres de soins vers Ipsogen, nous travaillons en partenariat avec des sous-traitants spécialisés dans le transport d'échantillons dans le cadre d'études multicentriques nationales ou internationales. Nos partenaires proposent un transport dédié en température contrôlée pour chaque type de prélèvement: transport en carboglace pour les échantillons congelés ou les sections de tissus sur lames histologiques, transport entre 15 et $25^{\circ} \mathrm{C}$ pour les blocs de tissus en paraffine, 4 à $8^{\circ} \mathrm{C}$ ou $-20^{\circ} \mathrm{C}$ pour les prélèvements en RNAlater ${ }^{\mathrm{TM}}$. Chaque expédition fait l'objet d'un soin particulier: suivi heure par heure, recharge en carboglace si nécessaire, utilisation d'une sonde de température possible pour les transits entre 4 et $8^{\circ} \mathrm{C} .$. . L'acheminement des échantillons se fait en 24 heures pour le réseau France et Europe, 48 heures depuis l'Amérique du nord et au maximun en 72 heures pour l'Asie et l'Australie, délais compatibles avec les exigences cliniques (rendu des résultats 3 semaines au plus après l'exérèse de la tumeur) dans le cadre des études prospectives.

\section{La randomisation}

Dans le cas des études rétrospectives concernant des échantillons archivés provenant de différents centres, nous procédons systématiquement à une randomisation des échantillons à chaque étape du processus. La randomisation a pour but de garantir la répartition aléatoire entre les échantillons de variables susceptibles d'interférer (biaiser) avec l'analyse de données. Elle permet de limiter le regroupement d'échantillons possédant des caractéristiques expérimentales communes (origine des échantillons, date de congélation, date d'extraction, jour de traitement de l'échantillon...) couramment rencontrés dans les technologies à grande échelle comme celle des puces à ADN.

\section{Le traitement des échantillons}

\section{Extraction des acides nucléiques}

La première étape du traitement des échantillons consiste à extraire les acides nucléiques ADN et ou ARN. S'il s'agit de tissus congelés, l'extraction des ARN et des ADN peut se faire simultanément sur gradient de chlorure de Césium. Cette méthode est également applicable pour les échantillons conservés en RNALater ${ }^{\mathrm{TM}}$. Bien que très efficace cette méthode de référence est assez lourde à mettre en œuvre: l'extraction se fait sur trois jours et comporte notamment une étape de centrifugation à très haute vitesse qui limite le débit d'extraction. Cette méthode est donc peu adaptée dans le cadre des études prospectives qui imposent un délai de 3 semaines au maximum entre l'exérèse et le rendu des résultats d'analyse. Nous recommandons dans ce cas l'utilisation de kits commerciaux (piagen par exemple) pour l'extraction des ARN ou des ADN.

Nous avons par ailleurs mis au point et validé des méthodes d'extraction des $A D N$ et des ARN après macro- ou micro-dissection au laser (laser capture microdissection, LCM) de tissus fixés inclus en paraffine. Selon la quantité de cellules disséquées, nous 
procédons à une étape d'amplification des ARN (amplification par transcription in vitro) ou des ADN (amplification du génome entier).

Dans tous les cas, les ARN sont qualifiés à l'aide du bioanalyseur 2100 d'Agilent qui permet d'évaluer le niveau de dégradation des ARN. Au-delà d'un certain seuil fixé de dégradation, l'échantillon est exclu de l'étude. L'intégrité des ADN est évaluée par $P C R$; les $A D N$ ne permettant pas d'amplifier des fragments de taille attendue sont exclus de I'analyse.

D'une manière générale, il est nécessaire d'homogénéiser et de standardiser les méthodes d'extraction au sein d'une même étude. Dans le cadre des études multicentriques, nous préconisons de centraliser cette étape afin de garantir une meilleure qualité des ARN en particulier, et éviter les biais expérimentaux liés à la méthode d'extraction employée, qui seraient critiques au moment de l'analyse des données.

\section{Analyses sur puces}

Plusieurs types de plates-formes pour l'étude des profils d'expression de gènes sont actuellement disponibles sur le marché. La plate-forme d'Ipsogen combine l'utilisation de puces très sensibles à grande échelle pour les études de développement et des puces dédiées à quelques centaines de gènes, issus des phases précédentes, pour les études de phase clinique. Les sondes préparées à partir des échantillons issus des phases de développement sont hybridées sur des puces à $A D N c$ DiscoveryChip ${ }^{T M}$ à 9600 gènes ou sur des puces à oligonucléotides pan-génomiques d'Affymetrix. Les puces pan-génomiques permettent un large criblage de gènes particulièrement adapté pour la phase «découverte » du développement du produit.

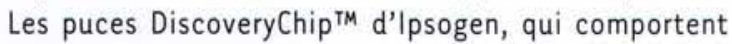
une grande majorité de gènes ayant un rôle connu ou putatif en oncogénèse, sont moins coûteuses et sont plus particulièrement désignées pour les phases de développement suivantes.

À cette étape encore, il est indispensable de standardiser le type de puces utilisées et les modes opératoires employés pour une même étude.

Un contrôle qualité (ARN de référence) est introduit systématiquement lors de chaque test de façon à s'assurer que le résultat reflète bien l'état biologique de l'échantillon, que la plateforme est toujours conforme et que les différents lots de puces et de réactifs présentent les mêmes performances que lors de la mise en place. Cet ARN de référence est utilisé comme "étalon 》 dans le cadre des études prospectives.

\section{Analyses par PCR en temps réel}

Si le nombre de biomarqueurs sélectionnés après la première phase de validation clinique ne dépasse pas la dizaine de gènes, la $P C R$ en temps réel peut être une alternative au système multiplex des puces à ADN. Notre technologie Profile Quant ${ }^{T M}$ permet de calibrer les mesures de PCR quantitative en temps réel afin de mesurer de manière absolue le nombre de copies d'un biomarqueur d'intérêt dans un échantillon (ARN extrait de tumeurs congelées ou en paraffine). Cette technologie s'appuie sur l'utilisation de standards plasmidiques contenant la séquence d'intérêt, qui sont fournis sous forme de dilutions en cascade. Ces gammes de dilutions permettent d'établir des courbes étalons; la quantification de l'échantillon inconnu est reportée sur la courbe étalon, et le signal correspondant est traduit en nombre de copies pour le biomarqueur étudié. Une normalisation par un gène de contrôle, ne variant pas dans les échantillons étudiés et mesuré grâce à la même technique, permet de s'affranchir des différences de quantités et de qualités d'ARN, d'efficacité de la transcription inverse etc.

\section{La gestion des données}

L'expertise d'Ipsogen en matière de bio-informatique et biostatistique est adaptée à l'analyse génomique des études pré-cliniques et cliniques. Notre système de gestion de données (LIMS, laboratory information management system) permet d'accéder aux données génomiques disponibles dans les bases publiques (Genbank, UniGene, SwissProt, Image, Locuslink, GO et Refseq), d'assurer la sauvegarde, la traçabilité et la gestion des données par projet, garantissant ainsi l'anonymat et la confidentialité dans un environnement BPF et BPL (Bonnes Pratiques de Fabrication et Bonnes Pratiques de Laboratoire) en conformité avec la réglementation américaine (FDA, Food and Drug Administration) concernant les enregistrements électroniques (21 (FR part 11).

\section{Conclusion}

Les analyses génomiques à grande échelle ont déjà permis d'identifier et de valider des biomarqueurs qui sont aujourd'hui utilisés dans le diagnostic de routine (ZAP70 dans les leucémies [12]). Les acteurs du développement des innovations thérapeutiques en oncologie prennent actuellement la mesure du potentiel des analyses génomiques: prises de décisions plus rationnelles dans le développement pharmaceutique en amont (toxicité, pharmacodynamique, mécanisme d'action), en phase clinique, possibilité de limiter les risques d'échec en enrichissant la population des répondeurs ou en évitant les patients développant des réactions toxiques. La mise en place d'une logistique adaptée à l'identification et la validation de biomarqueurs issus des technologies génomiques nécessite de maîtriser les technologies utilisées en aval, de l'analyse au traitement des données. Cette mise en place requiert une intégration forte de tous les acteurs impliqués: centres de soins, sociétés de biotechnologies et/ou industrie pharmaceutiques. $\diamond$ 


\section{SUMMARY}

Genomics and progress in oncology

Genomics and genetics are becoming pillars of clinical research, allowing certain diseases to be understood at the molecular level and leading to improved treatments. Successful genetic analyses in the clinical oncological setting depend on the quality of the samples collected. At clinical sites, attention must be paid to the steps taken prior to analysis, and to the fragility of the molecules to be tested. The development of a standard procedure specifically adapted to the identification and validation of biomarkers through genomics requires an understanding of the different technologies used at each time point, from the analysis to the data processing, and the implication of personnel at all levels : the health care centers, the biotech companies, and/or the pharmaceutical industries. $\Delta$

\section{RéFÉRENCES}

1. Schrohl AS, Holten-Andersen M, Sweep F, et al. Tumor markers: from laboratory to clinical utility. Mol Cell Proteomics $2003 ; 6: 378-87$.

2. Janne $P A$, Engelman JA, Johnson $B E$, et al. Epidermal growth factor receptor mutations in non-small-cell lung cancer: implications for treatment and tumor biology. J Clin Oncol 2005; $23: 3227-34$.

3. Pepe MS, Etzioni R, Feng Z, et al. Phases of biomarker development for early detection of cancer. J Natl Cancer Inst 2001 ; 93: 1054-61.
4. Department of Health and Human Services (HHS) Food and Drug Administration (FDA). Drug-diagnostic Co-Development concept paper, avril 2005.

5. Devilard $\varepsilon$, Bertucci $F$, Trempat $P$, et al. Gene expression profiling defines molecular subtypes of classical Hodgkin's disease. Oncogene 2002 ; 21: 3095-102.

6. Bertucci F, Nasser V, Granjeaud S, et al. Gene expression profiles of poorprognosis primary breast cancer correlate with survival. Hum Mol Genet $2002 ; 11: 863-72$.

7. Vey N, Mozziconacci MJ, Groulet-Martinec A, et al. Identification of new classes among acute myelogenous leukaemia with normal karyotype using gene expression profiling. Oncogene $2004 ; 23$; 9381-91.

8. Bertucci F, Borie N, Ginestier C, et al. Identification and validation of an ERBB2 gene expression signature in breast cancers. Oncogene 2004 ; $23: 2564-75$.

9. Bertucci F, Salas S, Eysteries S, et al. Gene expression profiling of colon cancer by DNA microarrays and correlation with histoclinical parameters. Oncogene $2004 ; 23: 1377-91$.

10. directive $98 / 79 \mathrm{CE}$. http://www. Ine. fr/publications/directives/98-79

11. Société Française de Pathologie, Société Française d'Hématologie, Société Française de Cancérologie, ANAES. Recommandations pour la cryopréservation de cellules et de tissus tumoraux dans le but de réaliser des analyses moléculaires.

12. Abbott BL. Advances in the diagnosis and treatment of chronic lymphocytic leukemia. Clin Adv Hematol Oncol $2004 ; 2$ : 448-54.

\section{TIRÉS À PART}

A. Groulet-Martinec 